\title{
An Optical MIM Pressure Sensor Based on a Double Square Ring Resonator
}

\author{
Pardis PALIZVAN $^{1}$, Saeed OLYAEE ${ }^{1 *}$, and Mahmood SEIFOURI ${ }^{2}$ \\ ${ }^{1}$ Nano-photonics and Optoelectronics Research Laboratory (NORLab), Shahid Rajaee Teacher Training University, \\ Lavizan 16788-15811, Tehran, Iran \\ ${ }^{2}$ Faculty of Electrical Engineering, Shahid Rajaee Teacher Training University, Lavizan 16788-15811, Tehran, Iran \\ *Corresponding author: Saeed OLYAEE_ Email: s_olyaee@sru.ac.ir
}

\begin{abstract}
In this paper, we have proposed a metal-insulator-metal (MIM) pressure sensor which consists of two plasmonic waveguides and a double square ring resonator. The two square rings are connected via a rectangular patch located between the two of them. The surface plasmon polaritons (SPPs) can be transferred from a square ring to the other through this patch. The finite-difference time-domain method (FDTD) has been used to simulate the device. Applying a pressure on the structure, it deforms, and a red shift of $103 \mathrm{~nm}$ in the resonance wavelength has been calculated. The deformation is linearly proportional to the wavelength shift in a wide range of wavelength. The proposed optical plasmonic pressure sensor has a sensitivity of $16.5 \mathrm{~nm} / \mathrm{MPa}$ which makes it very suitable for using in biological and biomedical engineering.
\end{abstract}

Keywords: Pressure sensor; plasmonic resonator; square ring resonator; MIM structure; surface plasmon polaritons

Citation: Pardis PALIZVAN, Saeed OLYAEE, and Mahmood SEIFOURI, "An Optical MIM Pressure Sensor Based on a Double Square Ring Resonator," Photonic Sensors, 2018, 8(3): 242-247.

\section{Introduction}

Metal-insulator-metal waveguides guide light via the refractive index differential between the insulating core and the conducting cladding. An effective characteristic in metal-insulator-metal (MIM) waveguides is that they guide optical modes in subwavelength scale regardless of plasmon resonance frequency and the field decay is partial in exterior areas of the waveguide [1]. In such structures, surface plasmon polaritons (SPPs) can simply be excited and confined in the insulator region. SPPs are electromagnetic waves which can be generated by illuminating a metal-dielectric interface [2]. MIM structures have been widely utilized in different devices like optical filters [3, 4], demultiplexers [5], sensors [6], and modulators [7] because of their strong localization, strong light confinement, and light manipulation at a subwavelength scale and high level of sensitivity to the surrounding environment features [8].

A pressure sensor is a device which can detect the applied pressure on it and responds by transforming the information to detectable electrical or optical signals. A positive aspect of optical pressure sensors is that they are immune to electromagnetic fields $[9,10]$. There have been several designs for optical pressure sensing devices such as all-fiber pressure sensor with $\mathrm{SiO}_{2}$ diaphragm proposed by Donlagic et al. [11], photonic-crystal fiber pressure sensor for dual environment monitoring by Osorio et al. [12], and 
fiber in-line Mach-Zehnder interferometer based on an inner air-cavity for high-pressure sensing proposed by Talataisong et al. [13].

Moreover, the use of different types of resonators in optical pressure sensor structures has been under a lot of attention due to their simple design and flexibility in selecting a desirable resonance wavelength. Olyaee et al. have proposed a photonic crystal cavity resonator by manipulating a two-dimensional photonic crystal structure in which the resonant wavelength is sensitive to the applied pressure with a sensitivity of $11.7 \mathrm{~nm} / \mathrm{GPa}$ [14-16]. $\mathrm{Wu}$ et al. also employed an H-type SPP resonator and MIM waveguides as a pressure sensor [17]. Zhao et al. designed a nano-opto-mechanical pressure sensor based on ring resonator which has a sensitivity of $1.47 \mathrm{pm} / \mathrm{kPa}$ [18].

Our research has been conducted on pressure sensing based on an MIM structure consisting of a double square ring resonator and SPP waveguides. The finite-difference time-domain (FDTD) method is employed to calculate the necessary characteristics. It is demonstrated that the resonant wavelength has a considerable shift when there is a deformation in the structure due to applied pressure.

\section{Device structure and the analysis method}

The structure of proposed device is illustrated in Fig. 1 in which the white parts represent air with dielectric permittivity of $\varepsilon_{d}=1$ and silver with permittivity of $\varepsilon_{s}$ is indicated in grey. The structure consists of an input and an output waveguides and two square resonators with a rectangular patch in the middle coupled with the two resonators. As the calculations show, the filtering characteristics like 3-dB bandwidth and peak-to-valley ratio are better for the filter with the dual-square resonator in comparison with a single square resonator filter [3]. In our structure, square resonators have outer length of $L_{1}$ and inner length of $L_{2}$ with respective values of $250 \mathrm{~nm}$ and $150 \mathrm{~nm}$, and they have a spacing of $g_{1}=60 \mathrm{~nm}$. The patch has dimensions of $w=20 \mathrm{~nm}$ and $h=50 \mathrm{~nm}$. The input and output waveguides are coupled to the square resonators with a gap width $g_{2}=10 \mathrm{~nm} . d_{1}$ which is the thickness of input, output, and waveguide (I) is equal to $50 \mathrm{~nm}$. Waveguide (I) is covered by a layer of silver which has a thickness of $t$ equal to $100 \mathrm{~nm}$. Applying a pressure on the silver layer, as shown in Fig. 1 inset, the waveguide (I) with a length of $L=700 \mathrm{~nm}$ deforms by the amount $d$.

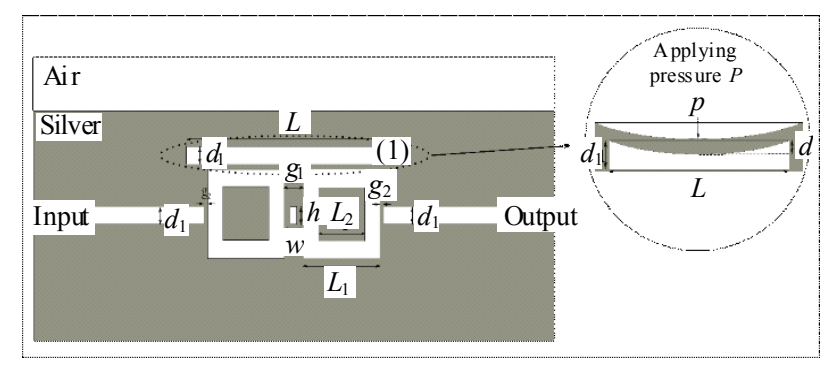

Fig. 1 Proposed device structure. The grey and white regions represent silver and air, respectively. The deformation $d$ of waveguide (I) under pressure $P$ is illustrated in the inset.

Plasmon waves in MIM waveguides have only TM components which are $E_{x}, H_{y}$, and $E_{z}$ while TE components are all zero [19]. Therefore, the dispersion relation of a plasmon slot waveguide is defined as [20]

$$
\tanh \left(\frac{k_{z 1} d}{2}\right)=-\frac{\varepsilon_{d} k_{z 2}}{\varepsilon_{s} k_{z 1}}
$$

where $d$ is the waveguide width, and the wave vectors $k_{z 1,2}$ are defined by momentum conservations as [21]

$$
k_{z 1,2}^{2}=\varepsilon_{d, s} k_{0}^{2}-\beta^{2}
$$

where $k_{0}=2 \pi / \lambda$ is the free space wave vector, and $\beta$ is the propagation constant of the MIM waveguide. $\varepsilon_{s}$ is the relative permittivity of silver and can be characterized by the Drude model as [22]

$$
\varepsilon_{s}(\omega)=\varepsilon_{\infty}-\frac{\omega_{n}^{2}}{\omega^{2}-\mathrm{i} \omega \Gamma_{0}}
$$

where $\omega$ is the angular frequency of the incident electromagnetic radiation, $\varepsilon_{\infty}=3.7$ is the relative permittivity at the infinite angular frequency, $\omega_{n}$ is the bulk plasma frequency equal to $1.38 \times 10^{16} \mathrm{~Hz}$, and finally $\Gamma_{0}=2.73 \times 10^{13} \mathrm{~Hz}$ is the oscillation damping of electrons.

When a TM polarized plane wave is launched to 
a metal-dielectric interface, a surface polarization is created by metal's electrons which provoke a localized electric field. In MIM waveguides, at each metal dielectric interface there are independent surface polarizations. Consequently, surface plasmons on the interface exhibit a beating frequency.

In our simulation, a plane wave is used to excite the surface plasmon polaritons in the input waveguide. The excited SPPs travel along the input waveguide and are coupled to the first square resonator. Then they are coupled to waveguide (I) and to the second square ring through the patch and finally to the output waveguide which is assumed to be long enough in order to make sure that the reflected waves are negligible. We have numerically calculated the resonance wavelength and have measured its shift while our structure is under pressure. The FDTD method with perfectly matched layer (PML) absorbing boundary conditions is employed to simulate the resonance characteristics of the proposed structure based on the pressure influence on the waveguide (I) volume. PML has been chosen so as to absorb light waves (propagating and evanescent) in the boundaries with zero reflection [23]. PML regions are divided into the minimum of 8 layers, and maximum of 8 layers, and the parameters are assumed to be $\alpha=0, \kappa=2$, and $\sigma=1$.

\section{Results and discussion}

The slight deformation in volume of waveguide (I) due to an applied pressure leads to a shift in resonant wavelength of the structure, since the light propagating in the resonators is coupled to the waveguide (I) on its way to the output waveguide. The relationship between the resonant frequency shift and the change in the resonating volume can be defined by Slater's law as [4]

$$
\frac{\delta f}{f}=-\frac{\left(\varepsilon_{0} E^{2}-\mu_{0} H^{2}\right) \delta V}{\int_{V}\left(\varepsilon_{0} E^{2}+\mu_{0} H^{2}\right) d V}
$$

where $E$ and $H$ are electric and magnetic fields, and $f$ is the resonant frequency of resonating area with a volume of $V$. According to this law, by a change in volume the resonant wavelength shifts [24].

As shown in Fig. 2, our structure has three resonance wavelengths in res $1=419.9 \mathrm{~nm}$, res2 $=$ $520 \mathrm{~nm}$, and res $3=970 \mathrm{~nm}$ without any applied pressure. But when there is a $10 \mathrm{~nm}$ deformation in waveguide (I) corresponding to $6.2 \mathrm{MPa}$ pressure, the resonance wavelengths have been respectively shifted to $420.2 \mathrm{~nm}, 534 \mathrm{~nm}$, and $1073 \mathrm{~nm}$. The latter resonance wavelength (res3) has a shift of $103 \mathrm{~nm}$ while the others (res1 \& res2) have $0.3 \mathrm{~nm}$ and $14 \mathrm{~nm}$ red shifts. Obviously, the third resonance wavelength res $3=970 \mathrm{~nm}$ is shifted more than the other ones. Therefore, the following calculations are all based on the third resonance peak.

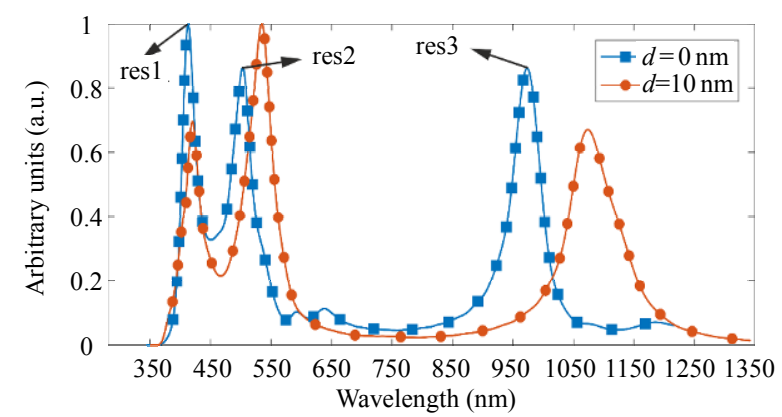

Fig. 2 Resonance peaks of the proposed structure without pressure $(d=0 \mathrm{~nm})$ and under pressure $(d=10 \mathrm{~nm})$.

The patch coupled to two square resonators makes the $3 \mathrm{~dB}$ bandwidth of the resonant wavelengths thinner. Moreover, without the patch in this structure the two square resonators will be directly coupled to each other which can lead to splits in resonant peaks [3].

In order to have a better understanding of the resonance in the structure, the contour profiles of the field $H_{y}$ for three different wavelengths without any pressure on the structure are illustrated in Fig. 3. Figure 3(a) shows the second resonance (res2) and the way it is resonating. In Fig. 3(b), most of the light seems to reflect back to the input port as it is obvious from Fig. 2 that the wavelength of $726 \mathrm{~nm}$ is not a resonance wavelength so it cannot pass through the structure. Additionally, Fig. 3(c) demonstrates the field $H_{y}$ of almost resonating wavelength of $996 \mathrm{~nm}$. 


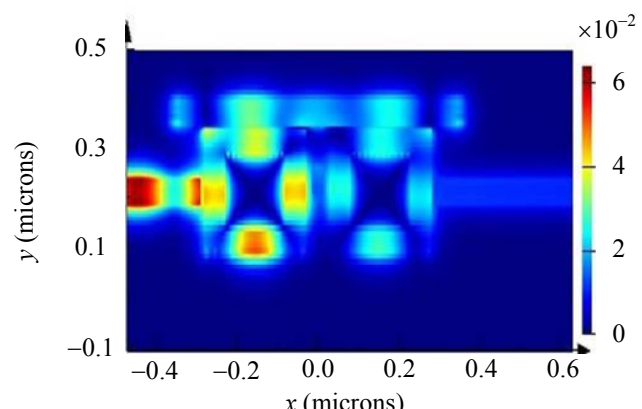

(a)

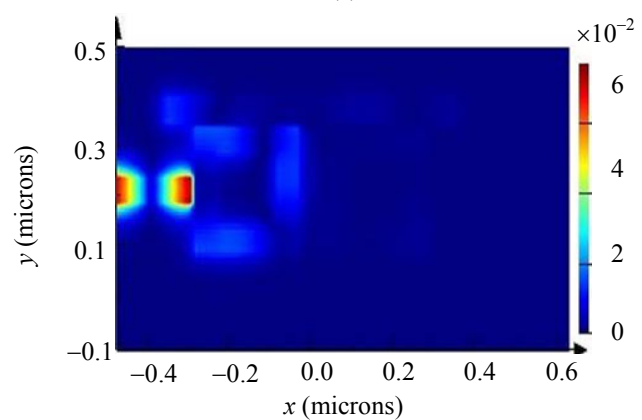

(b)

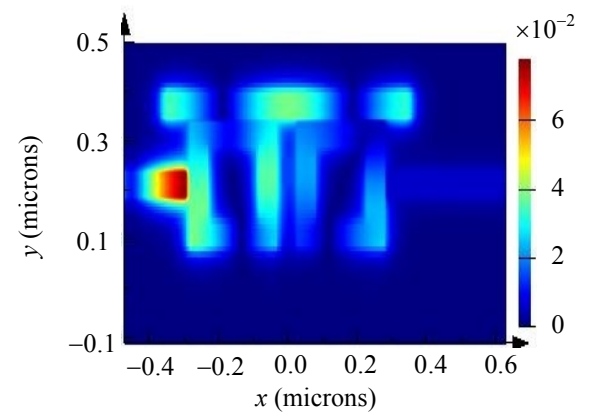

(c)

Fig. 3 Contour profiles of the H-fields for three wavelengths at (a) $520 \mathrm{~nm}$, (b) $726 \mathrm{~nm}$, and (c) $996 \mathrm{~nm}$ with no pressure.

We have computed the resonance wavelength shift based on the waveguide (I) deformation (d). As deformation increases by $2 \mathrm{~nm}$, the resonance wavelength has approximately $20-\mathrm{nm}$ red shift as shown in Fig.4, except for the deformation equals to $2 \mathrm{~nm}$. The $20-\mathrm{nm}$ shift corresponding to $2-\mathrm{nm}$ deformation of waveguide (I) is more than the same previous works and is a promising feature in these types of sensors [17, 20].

The relationship between the wavelength shift and six different deformations is demonstrated in Fig. 5 which is quietly linear with regression coefficient of $R^{2}=0.9751$, hence it can be utilized to estimate our sensors performance and their sensitivity.

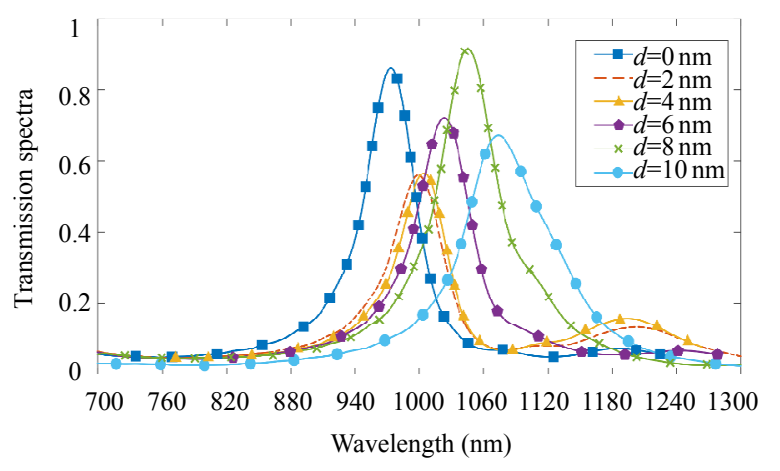

Fig. 4 Transmission spectra for different deformations $d$ of the res3.

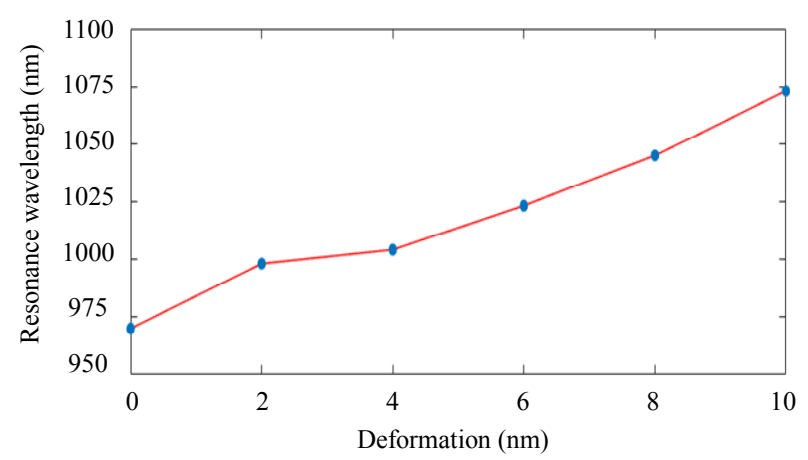

Fig. 5 Resonance wavelength as a function of the deformation of waveguide (I).

The pressure influence on the slab waveguide (I) has been called deformation. Considering the top silver layer a plane plate with the length of $L$ and thickness of $t$, the relation between pressure and deformation (d) and consequently the wavelength shift can be calculated as [17]

$$
P=\frac{2 Y t^{3} d}{L^{4}}
$$

where $P$ is the pressure applied on the sensor, and $Y$ $=7.5 \times 10^{10} \mathrm{~Pa}$ is the Young's modulus of silver. As it is shown in Fig. 5 , wavelength shift $\Delta \lambda$ is related to deformation $(d)$ of the resonator with a coefficient of $\alpha$ so the equation can be obtained as follows:

$$
P=\frac{2 Y t^{3}}{\alpha L^{4}} \Delta \lambda .
$$

Sensitivity $s$ is another important parameter in sensor [25]. According to that, the sensitivity of the proposed sensor is defined by (7), and considering the parameters in our paper, the sensitivity is as high as $16.5 \mathrm{~nm} / \mathrm{MPa}$.

$$
s=\frac{\Delta \lambda}{\Delta P}=\frac{L^{4} \Delta \lambda}{2 Y t^{3} \Delta d} .
$$


According to the mentioned equations and the results of similar previous works, a comparison is listed in Table 1.

Table 1 Comparison between our structure and the previous ones in terms of sensitivity and resonance wavelength shift.

\begin{tabular}{ccc}
\hline SPP pressure sensor structure & Sensitivity $(\mathrm{nm} / \mathrm{MPa})$ & $\Delta \lambda(\mathrm{nm})$ \\
\hline H-type resonator [17] & 5.12 & $32 \mathrm{~nm}$ \\
$\pi$-shape resonator [20] & 8.5 & $80 \mathrm{~nm}$ \\
Double square resonator & 16.5 & $103 \mathrm{~nm}$ \\
\hline
\end{tabular}

\section{Conclusions}

In conclusions, we have proposed an MIM structure including a double square resonator and plasmonic waveguides. The resonance wavelengths are calculated by the FDTD method. The simulation results show that by increasing the deformation in the resonator, a red shift occurs in the resonance wavelength and the relationship between the shift and deformation is almost linear. The relationship between the deformation and pressure has been discussed as well as the sensitivity of the proposed sensor, which is about $16.5 \mathrm{~nm} / \mathrm{MPa}$. This pressure sensor structure may have potential applications in nano optical plasmonic-based devices. Moreover, despite the nano-scale dimensions of the presented sensor, it can be fabricated due to the rectangular measurements of waveguides and resonators.

\section{Acknowledgment}

This work was supported by Shahid Rajaee Teacher Training University under Grant No. 3359.

Open Access This article is distributed under the terms of the Creative Commons Attribution 4.0 International License (http://creativecommons.org/licenses/by/4.0/), which permits unrestricted use, distribution, and reproduction in any medium, provided you give appropriate credit to the original author(s) and the source, provide a link to the Creative Commons license, and indicate if changes were made.

\section{References}

[1] A. Polman, "Plasmon slot waveguides: towards chip-scale propagation with subwavelength-scale localization," Physical Review B, 2006, 73(3): 1-9.

[2] A. A. Maradudin, Introduction: plasmonics and its building blocks. Amsterdam, Netherlands: Elsevier B. V., 2014: 1-36.

[3] G. Y. Duan, P. L. Lang, L. L. Wang, L. Yu, and J. H. Xiao, "A band-pass plasmonic filter with dual-square ring resonator," Modern Physics Letters B, 2014, 28(23): 1450188-1-1450188-8.

[4] H. Q. Wang, J. B. Yang, J. J. Zhang, J. Huang, W. J. $\mathrm{Wu}, \mathrm{D}$. B. Chen, et al., "Tunable band-stop plasmonic waveguide filter with symmetrical multiple-teeth-shaped structure," Optical Letters, 2016, 41(3): 1233-1236.

[5] M. R. Rakhshani and M. A. Mansouri-birjandi, "Dual wavelength demultiplexer based on metalinsulator-metal plasmonic circular ring resonators," Journal of Modern Optics, 2016, 63(11): 1078-1086.

[6] M. R. Rakhshani and M. A. Mansouri-birjandi, "High sensitivity plasmonic sensor based on metal-insulatormetal waveguide and hexagonal-ring cavity," IEEE Sensors Journal, 2016, 16(9): 3041-3046.

[7] X. Piao, S. Yu, and N. Park, "Control of Fano asymmetry in plasmon induced transparency and its application to plasmonic waveguide modulator," Optics Express, 2012, 20(17): 18994-18999.

[8] H. Lu, X. M. Liu, Y. K. Gong, D. Mao, and G. X. Wang, "Analysis of nanoplasmonic wavelength demultiplexing based on metal-insulator-metal waveguides," Journal of the Optical Society of America B, 2011, 28(7): 1616-1621.

[9] C. Paper, S. Upadhyay, J. Foundation, V. Laxmi, K. Govt, and C. C. Govt, "Designing and optimization of nano-ring resonator-based photonic pressure sensor," in Proceeding of International Conference on Information and Communication Technology for Sustainable Development (ICT4SD), Ahmedabad, India, 2016, pp. 269-278.

[10] M. C. Oh, J. W. Kim, K. J. Kim, and S. S. Lee, "Optical pressure sensors based on vertical directional coupling with flexible polymer waveguides," IEEE Journals \& Magazines, 2009, 21(8): 501-503.

[11] D. Donlagic and E. Cibula, "All-fiber high-sensitivity pressure sensor with $\mathrm{SiO}_{2}$ diaphragm," Optics Letters, 2005, 30(16): 2071-2073.

[12] J. H. Osório, J. G. Hayashi, Y. A. V Espinel, M. A. R. Franco, M. V Andrés, and C. M. B. Cordeiro, "Photonic-crystal fiber-based pressure sensor for dual environment monitoring," Applied Optics, 2014, 53(17): 3668-3672.

[13] W. Talataisong, D. N. Wang, R. Chitaree, C. R. Liao, and C. Wang, "Fiber in-line Mach-Zehnder interferometer based on an inner air-cavity for high-pressure sensing," Optics Letters, 2015, 40(7): $1220-1222$ 
[14] S. Olyaee and A. A. Dehghani, "High resolution and wide dynamic range pressure sensor based on two-dimensional photonic crystal," Photonic Sensors, 2012, 2(1): 92-96.

[15] S. Olyaee and A. A. Dehghani, "Ultrasensitive pressure sensor based on point defect resonant cavity in photonic crystal," Sensor Letters, 2013, 11(10): 1854-1859.

[16] S. Olyaee and A. A. Dehghani, "Nano-pressure sensor using high quality photonic crystal cavity pesonator," in Proceeding of IET 8th International Symposium on Communication Systems, Networks and Digital Signal Processing (CSNDSP 2012), Poznań, Poland, 2012, pp. 1-4.

[17] J. Wu, P. L. Lang, X. Chen, and R. Zhang, "A novel optical pressure sensor based on surface plasmon polariton resonator," Journal of Modern Optics, 2015, 63(3): 219-223.

[18] X. Zhao, J. M. Tsai, H. Cai, X. M. Ji, and J. Zhou, "A nano-opto-mechanical pressure sensor via ring resonator," Optics Express, 2012, 20(8): 8535-8542.

[19] B. L. J. Park and H. Kim, "High order plasmonic Bragg reflection in the metal-insulator-metal waveguide Bragg grating," Optics Express, 2008, 16(1): 413-425.
[20] G. Y. Duan, P. L. Lang, L. L. Wang, L. Yu, and J. H. Xiao, "An optical pressure sensor based on $\pi$-shaped surface plasmon polariton resonator," Modern Physics Letters B, 2016, 30(21): 1650284-1-1650284-8.

[21] T. S. Wu, Y. M. Liu, Z. Y. Yu, H. Ye, C. G. Shu, Y. W. Peng, et al., "Tuning the Fano resonances in a single defect nanocavity coupled with a plasmonic waveguide for sensing applications," Modern Physics Letters B, 2015, 29(33): 1550218-1-1550218-10.

[22] J. Liu, G. Fang, H. Zhao, and Y. Zhang, "Plasmon flow control at gap waveguide junctions using square ring resonators," Journal of Physics D Applied Physics, 2010, 43(5): 55103-1-55103-6.

[23] J. Bérenger, "Perfectly matched layer (PML) for computational electromagnetics," Synthesis Lectures on Computational Electromagnetics, 2007, 2(1): $1-117$.

[24] L. C. Maier and J. C. Slater, "Field strength measurements in resonant cavities," Journal of Applied Physics, 1952, 23(1): 68-77.

[25] Z. Zalevsky and I. Abdulhalim, Integrated nanophotonic devices. Amsterdam, Netherlands: Elsevier B.V., 2014: 1-274. 